Am J Prev Med. 2019 June ; 56(6): 834-843. doi:10.1016/j.amepre.2019.01.003.

\title{
Prevalence and Correlates of Receiving Medical Advice to Increase Physical Activity in U.S. Adults: National Health and Nutrition Examination Survey 2013-2016
}

\author{
Marissa L. Zwald, PhD, MPH ${ }^{1,2}$, Brian K. Kit, MD, MPH ${ }^{2,3}$, Tala H.I. Fakhouri, PhD, MPH ${ }^{1}$, \\ Jeff P. Hughes, MPH $^{1}$, Lara J. Akinbami, MD ${ }^{1,2}$ \\ ${ }^{1}$ Division of Health and Nutrition Examination Surveys, National Center for Health Statistics, \\ Centers for Disease Control and Prevention, Hyattsville, Maryland ${ }^{2}$ U.S. Public Health Service, \\ Rockville, Maryland ${ }^{3} \mathrm{NIH}$, Bethesda, Maryland
}

\begin{abstract}
Introduction: National objectives recommend healthcare professionals provide physical activity advice. This study examined health and demographic characteristics associated with receipt of medical advice to increase physical activity among U.S. health care-utilizing adults and differences in associations by age group.
\end{abstract}

\begin{abstract}
Methods: Analyses included 8,410 health care-utilizing adults aged $\geq 20$ years from the 20132016 National Health and Nutrition Examination Surveys (analyzed in 2018). Logistic regression was used to examine associations between receipt of medical advice to increase physical activity in the past year and measured health conditions, reported health behaviors, and demographic characteristics. Models were stratified by age group (20-39, 40-59, and $\geq 60$ years).
\end{abstract}

Results: Physical activity medical advice was received by $42.9 \%(95 \% \mathrm{CI}=40.8,44.9)$ of adults overall. By age group, $32.7 \%$ of younger adults, $46.7 \%$ of middle-aged adults, and $48.9 \%$ of older adults received advice. Among all adults and across all age groups, receipt of advice was higher among adults with chronic health conditions: obesity $(63.0 \%, 95 \% \mathrm{CI}=60.3,65.7)$, hypertension $(56.5 \%, 95 \%=$ CI $53.8,59.2)$, diabetes $(69.8 \%, 95 \% \mathrm{CI}=66.5,72.8)$, hypercholesterolemia $(55.6 \%$, $95 \% \mathrm{CI}=52.3,59.0)$, and low high-density lipoprotein cholesterol $(53.8 \%, 95 \% \mathrm{CI}=50.1,57.4)$. Among all adults, those with obesity, hypertension, and diabetes had significantly greater odds of receipt of advice after adjustment. Stronger associations between diabetes and hypercholesterolemia and receiving physical activity advice were observed among younger adults.

Conclusions: Receipt of physical activity medical advice was highest among adults with specific chronic health conditions, and this pattern was stronger among younger adults with

\footnotetext{
Address correspondence to: Marissa L. Zwald, PhD, MPH, Division of Health and Nutrition Examination Surveys, National Center for Health Statistics, Centers for Disease Control and Prevention, 4770 Buford Highway NE, Atlanta GA 30341. mzwald@cdc.gov. No financial disclosures were reported by the authors of this paper. SUPPLEMENTAL MATERIAL

Supplemental materials associated with this article can be found in the online version at https://doi.org/10.1016/j.amepre.2019.01.003.
} 
diabetes and hypercholesterolemia. However, most health care-utilizing adults did not receive physical activity medical advice.

\section{INTRODUCTION}

National health objectives recommend that healthcare professionals provide physical activity (PA) counseling and education to their patients, particularly those with chronic diseases. ${ }^{1-4}$ One objective outlined in Healthy People 2020 is to increase the proportion of office visits made by patients with a diagnosis of cardiovascular disease, diabetes, or hyperlipidemia that include counseling or education related to exercise. ${ }^{1}$ Another objective described in the U.S. National Physical Activity Plan is for healthcare systems to increase the priority of PA assessment, advice, and promotion. ${ }^{2,5}$ Additionally, the American College of Preventive Medicine calls upon primary care providers to incorporate PA counseling into routine patient visits, stating effective interventions can be as brief as 2-4 minutes, but suggesting longer sessions should be used, depending on the needs and characteristics of patients. ${ }^{3}$ Other professional organizations have released position statements that have addressed the importance of PA counseling, including the American Academy of Family Physicians, the American College of Sports Medicine, and the American Heart Association. ${ }^{6-9}$ Despite these national priorities and recommendations, there is limited recent information on the association between patient health and demographic characteristics and receiving PA counseling in healthcare settings. ${ }^{10-12}$ Therefore, the aim of the current study is to examine health and demographic characteristics of U.S. health care-utilizing adults associated with receipt of medical advice to increase their PA. Because previous research has shown medical advice to exercise is often reported within specific age groups, particularly among middleand older-aged adults, ${ }^{13}$ this study also seeks to examine differences by age group in associations between characteristics and receipt of medical advice to increase PA.

\section{METHODS}

Data from the most recently available 4-year cycle (2013-2016) of National Health and Nutrition Examination Survey (NHANES), a multipurpose health survey, were used. The survey used a complex sampling design to produce a nationally representative sample of the U.S. civilian non-institutionalized population. The survey included interviews conducted in the home and physical assessments, including the collection of blood samples and measurement of blood pressure, height, and weight, in a mobile examination center. The National Center for Health Statistics Research Ethics Review Board approved the protocol and informed consent was obtained from all participants. The overall examination response rate for adults aged $\geq 20$ years for $2013-2016$ was $61.9 \% .{ }^{14}$ Additional NHANES details are available elsewhere. ${ }^{15-18}$

\section{Study Sample}

In NHANES survey cycles 2013-2016, a total of 11,062 adults aged $\geq 20$ years were interviewed and examined (Figure 1). Pregnant women ( $n=135)$ were excluded from this analysis. The analysis was also restricted to those who utilized healthcare services in the past year, defined as seeing or talking to a healthcare provider in the previous 12 months. Therefore, non-health care-utilizing adults who did not have an opportunity to receive 
advice were excluded $(n=1,815)$, resulting in an eligible sample of 9,112 adults. Participants missing data for the outcome variable of receipt of PA medical advice $(n=3)$ or any health conditions ( $n=699,7.7 \%$ missing from analytic sample) were further excluded from analyses, resulting in a final analytic sample of 8,410 adults.

\section{Measures}

The outcome of interest was receipt of medical advice to increase PA. All adults participating in the NHANES home interview, regardless of reported health conditions or diseases, were asked: To lower your risk for certain diseases, during the last 12 months have you ever been told by a doctor or health professional to increase your PA or exercise? Response options were yes or no. ${ }^{13}$

To examine associations between medical advice to increase PA and health conditions, health behaviors, and demographic characteristics, the following covariates were included in the analysis. Health conditions, which were selected based on prior evidence demonstrating beneficial associations between PA and each objectively measured chronic health condition, included: obesity, hypertension, diabetes, hypercholesterolemia, and low high-density lipoprotein (HDL) cholesterol. ${ }^{19}$ Obesity was defined as BMI (measured weight in kilograms divided by measured height in meters squared) $\geq 30$. Hypertension was defined as measured systolic blood pressure $\geq 140 \mathrm{mmHg}$, diastolic blood pressure $\geq 90 \mathrm{mmHg}$, or selfreported current use of hypertension medication, according to hypertension guideline recommendations in effect through $2016 .{ }^{20}$ Diabetes was defined as HbA1c $\Varangle 6.5 \%$ or selfreported physician diagnosis of diabetes. Hypercholesterolemia was defined as serum total cholesterol $\geq 240 \mathrm{mg} / \mathrm{dL}$ or self-reported current use of cholesterol-lowering medication. Low HDL cholesterol was defined as serum HDL cholesterol $<40 \mathrm{mg} / \mathrm{dL}$.

Self-reported health behaviors, which were based on previous research, ${ }^{11,12}$ included: smoking status (never smoked, former smoker, current smoker) and adherence to the national PA aerobic guidelines of the equivalent of $\geq 150$ minutes of moderate-intensity activity in the previous week (met PA guidelines or did not meet PA guidelines). ${ }^{21}$

Demographic characteristics (selected a priori and based on factors described in the literature that may influence patient and provider communication ${ }^{22-24}$ ) included: age group (20-39, 40-59, 260 years), sex (male or female), race/Hispanic origin (Hispanic, nonHispanic white, non-Hispanic black, non-Hispanic Asian, and other, which included all nonHispanic individuals who reported more than one race group), educational level (less than high school, high school, some college, college or more), poverty status based on family income-to-poverty ratio (FIPR, calculated by dividing family income by the U.S. poverty threshold specific for family size and where categories were selected based on Supplemental Nutrition Assistance Program income eligibility limits; <130\% FIPR, 130\%-349\% FIPR, or $2350 \%$ FIPR), ${ }^{25}$ and health insurance status (whether participant was or was not covered by health insurance or some other kind of healthcare plan).

\section{Statistical Analysis}

SAS, version 9.4 and SUDAAN, version 11.0.1 were used to conduct analyses to account for the complex sampling design. Examination sampling weights were used. Prevalence 
estimates and 95\% CIs of receipt of PA medical advice are reported for all participants and for each age group separately. Multivariate logistic regression was used to examine health and demographic characteristics (measured health conditions, smoking status, adherence to the national PA guidelines, age group, sex, race/Hispanic origin, educational level, poverty status, and health insurance status) associated with receiving PA medical advice. The adjusted model included all covariates, except for age group. There was no evidence of multicollinearity between health conditions after examining the SEs for each health condition in models including and excluding all other health conditions. Therefore, all health conditions were included in a single model. Finally, differences in associations between health and demographic characteristics and receipt of PA medical advice were assessed by age group by including interaction terms between age group $\mathrm{X}$ each characteristic in the model. Each interaction term was entered and evaluated separately.

Preliminary analyses demonstrated that interaction terms between age group $\mathrm{X}$ three of the health conditions and behaviors assessed (diabetes [ $p=0.016$ ], hypercholesterolemia $[p=0.026]$, and smoking status [ $p=0.026])$ were significant. No interaction terms between age group $\mathrm{X}$ demographic characteristics were significant. Although most interactions with age group were not significant, results are presented stratified due to the clinical relevance of age group and receipt of PA medical advice. ${ }^{26}$

Because $\cong 8 \%$ of the examined sample had missing data for the health conditions investigated in this study, data were reweighted to assess the impact of excluding these participants using the PROC WTADJUST command in SUDAAN. The adjusted sample weights produced similar results to those estimated with the publicly available examination sample weights. Thus, results obtained with the use of the publicly available examination sample weights are presented here. Characteristics for adults excluded because of missing data for health conditions are compared with the final sample in Appendix Table 1 (available online). Data analysis occurred in 2018.

\section{RESULTS}

Approximately $43 \%(95 \% \mathrm{CI}=40.8,44.9)$ of health care-utilizing adults reported that their doctor or another healthcare professional advised them to increase their PA (Table 1), whereas $57.1 \%$ (95\% CI=55.1, 59.2) did not receive PA medical advice (data not shown). The prevalence of receiving PA medical advice was higher among adults with chronic health conditions, including obesity $(63.0 \%, 95 \% \mathrm{CI}=60.3,65.7)$, hypertension $(56.5 \%, 95 \%$ $\mathrm{CI}=53.8,59.2)$, diabetes $(69.8 \%, 95 \% \mathrm{CI}=66.5,72.8)$, hypercholesterolemia $(55.6 \%, 95 \%$ $\mathrm{CI}=52.3,59.0)$, and low HDL cholesterol $(53.8 \%, 95 \% \mathrm{CI}=50.1,57.4)$ compared with those without the conditions. Among all adults without each individual chronic health condition, receipt of PA medical advice ranged from $\cong 30 \%$ to $40 \%$. Additionally, receiving PA medical advice was reported by a higher proportion of health care-utilizing adults who identified as female, aged $\succ 60$ years, Hispanic or non-Hispanic black, had some college education, had health insurance, and reported being a former smoker and did not meet national PA guidelines. By age group, the prevalence of receipt of PA medical advice was 32.7\% among younger adults $(95 \% \mathrm{CI}=30.7,34.8), 46.7 \%(95 \% \mathrm{CI}=43.2,50.2)$ among middle-aged adults, and $48.9 \%$ (95\% CI=46.2, 51.5) among older-aged adults. 
Adjusted multivariate logistic regression results for all age groups combined and stratified by age group are presented in Table 2. After adjustment for all other health and demographic characteristics, compared with adults without each health condition, those with obesity $(\mathrm{AOR}=3.36,95 \% \mathrm{CI}=2.85,3.97)$, hypertension $(\mathrm{AOR}=1.54,95 \% \mathrm{CI}=1.31,1.80)$, diabetes $(\mathrm{AOR}=2.03,95 \% \mathrm{CI}=1.67,2.48)$, hypercholesterolemia $(\mathrm{AOR}=1.52,95 \% \mathrm{CI}=1.26,1.82)$, and low HDL cholesterol $(\mathrm{AOR}=1.34,95 \% \mathrm{CI}=1.12,1.62)$ had greater odds of reporting receipt of advice from a healthcare professional to increase their PA. Significant associations between selected health behaviors $\mathrm{X}$ receipt of PA medical advice were observed among all adults. Compared with all adults who never smoked, current smokers had greater odds of receiving $\mathrm{PA}$ medical advice $(\mathrm{AOR}=1.20,95 \% \mathrm{CI}=1.02,1.40)$. The odds of receiving PA medical advice among former smokers compared with nonsmokers was similar, although the $95 \% \mathrm{CI}$ included the value of $1(\mathrm{AOR}=1.23,95 \% \mathrm{CI}=0.99,1.54)$. Not meeting the national PA guidelines was associated with receiving advice (versus meeting the guidelines; $\mathrm{AOR}=1.28,95 \% \mathrm{CI}=1.12,1.45)$.

Associations between receipt of PA advice and demographic characteristics varied by subgroup (Table 2). Those aged 40-59 years had greater odds of receiving PA medical advice than adults aged $20-39$ years ( $\mathrm{AOR}=1.24,95 \% \mathrm{CI}=1.07,1.43$ ). No significant differences were observed between older adults ( $\ 60$ years) and adults aged 20-39 years $(\mathrm{AOR}=1.08,95 \% \mathrm{CI}=0.91,1.28)$. Female adults had greater odds of receiving advice than male adults $(\mathrm{AOR}=1.25,95 \% \mathrm{CI}=1.09,1.43)$. Adults who identified as Hispanic $(\mathrm{AOR}=1.82,95 \% \mathrm{CI}=1.48,2.24)$, non-Hispanic black ( $\mathrm{AOR}=1.22,95 \% \mathrm{CI}=1.01,1.47)$, and non-Hispanic Asian ( $\mathrm{AOR}=1.48,95 \% \mathrm{CI}=1.21,1.81$ ) had greater odds of receiving PA medical advice than non-Hispanic whites. Compared with adults with a college degree or higher, adults with less than a high school education ( $\mathrm{AOR}=0.75,95 \% \mathrm{CI}=0.60,0.94)$ or a high school education only ( $\mathrm{AOR}=0.85,95 \% \mathrm{CI}=0.73,0.99$ ) had lesser odds of receiving PA medical advice. Compared with adults within the highest income category, adults among the lowest income category had lesser odds of receiving advice ( $\mathrm{AOR}=0.84,95 \% \mathrm{CI}=0.72$, 0.99). Adults without health insurance had lesser odds of receiving medical advice than insured adults $(\mathrm{AOR}=0.73,95 \% \mathrm{CI}=0.59,0.90)$.

Significant interactions between age group $X$ three of the health characteristics assessed, including diabetes $(p=0.016)$, hypercholesterolemia $(p=0.026)$, and smoking status ( $p=0.026$ ), were observed, and adjusted multivariate logistic regression results stratified by age group are also shown in Table 2. Stronger associations between diabetes and hypercholesterolemia and receiving PA advice were observed among younger adults. The AOR for the association between diabetes $\mathrm{X}$ advice was 3.18 (95\% CI=1.86, 5.44) for those aged 20-39 years; 2.47 (95\% CI=1.68, 3.64) for those aged 40-59 years; and 1.64 (95\% $\mathrm{CI}=1.23,2.20$ ) for those aged $\succeq 0$ years. A similar pattern was observed for hypercholesterolemia (20-39 years: $\mathrm{AOR}=1.64,95 \% \mathrm{CI}=1.11,2.43,40-59$ years: $\mathrm{AOR}=1.78,95 \% \mathrm{CI}=1.39,2.27$, and $\succ 60$ years: $\mathrm{AOR}=1.25,95 \% \mathrm{CI}=0.97,1.62)$. Stronger associations among younger adults, compared with older adults, were also observed for smoking status. 


\section{DISCUSSION}

Increased evidence demonstrates the importance of PA promotion within healthcare settings and recommendations from several professional organizations encourage PA counseling by healthcare providers. ${ }^{27-35}$ The present study's findings suggest that approximately $43 \%$ of U.S. health care-utilizing adults reported receiving medical advice to increase PA in 20132016, and approximately $60 \%-70 \%$ of adults with obesity, hypertension, diabetes, hypercholesterolemia, and low HDL cholesterol received PA medical advice. Similar to the results of previous studies, ${ }^{11,12}$ receipt of PA medical advice was more commonly reported among those with chronic diseases than those without.

As reported in prior research, the percentage of adults who received PA medical advice increased with older age groups. ${ }^{13}$ To the authors' knowledge, age group differences in the associations between health characteristics and PA medical advice have not been investigated previously. Despite the lower prevalence of PA medical advice among younger adults, findings in this study suggested that the associations between chronic health conditions and receipt of PA medical advice were generally stronger among those in this younger age group; however, only diabetes and hypercholesterolemia had a significant interaction with age. Health conditions perceived to be associated with advanced age may be more likely to trigger healthcare professionals to provide PA advice when they are present among younger adults compared with younger adults without these health conditions. By contrast, people with obesity had more uniform odds of receipt of PA medical advice across age groups, and there was not a significant interaction with age.

Independent of health status and health behaviors, the associations between receipt of PA medical advice and certain demographic characteristics, including sex and race/Hispanic origin, were consistent with results highlighted in past studies that women (versus men) and non-white adults (compared with white adults) have greater odds of receiving PA medical advice. ${ }^{10-12,36}$ Existing clinical recommendations suggest healthcare providers tailor PA medical advice (i.e., content of the counseling, behavioral change approach used, and delivery method of advice) to certain patient subgroups based on some of these aforementioned sociodemographic characteristics, in addition to existing health conditions and current health behaviors. ${ }^{3}$ Previous research has also demonstrated that low education levels, low income, and lack of health insurance are associated with a decreased odds of receiving PA medical advice, ${ }^{11}$ and this study found similar patterns. Further research may help to disentangle barriers to receipt of PA medical advice among patients with these specific characteristics. Such barriers identified in previous literature might include competing health concerns during medical visits, limited access to ongoing preventive health care, and lower perceived opportunity and resources to engage in PA, in addition to healthcare provider time demands, limited training and expertise in the benefits of PA and behavioral change, limited perceived efficacy, and limited support systems for patient education. 3,5,11,33,37-42 In addition to research on potential barriers, future analyses could ascertain if there are specific demographic and health characteristics associated with not receiving advice, specifically within the subgroups that this study identified as likely to be advised about PA, including those with chronic health conditions. 
Past reviews of the literature have reported public health implications of PA medical advice. 3,4,39,41 This includes potential benefits associated with reduced cardiovascular disease risk among adult patients with and without chronic health conditions, as well as potential harms, including falls, injuries, and cardiovascular disease-related events. ${ }^{39,41}$ Future researchers might examine additional downstream effects of PA medical advice using varied health measures, as well as the economic implications of each of those effects. ${ }^{5,26,43}$ Additionally, although the present study focused on examining the health and demographic characteristics associated with PA medical advice, more research on the correlates of medical advice promoting other types of health behaviors, such as controlling or maintaining body weight, incorporating a healthful diet, or reducing sedentary behaviors, may aid public health and healthcare professionals. ${ }^{4,39,41}$

\section{Limitations}

The current study has several limitations. Self-reported data collected from the PA medical advice measure and the PA measure used may be subject to recall or social desirability bias.

44 The PA medical advice measure used only assessed whether an individual received advice from a healthcare provider to increase PA, and not medical advice to sustain or decrease PA levels. Additionally, the specificity of the PA medical advice measure, which includes an introductory prompt that asks participants about their receipt of PA medical advice in order to lower their risk for certain diseases, may have influenced participant responses.

Specifically, although the question is asked of all adults participating in the NHANES home interview regardless of their health status, those with a chronic health condition may have interpreted and answered the question differently from those without a chronic health condition. For some of the health conditions assessed in this study, no information was available about whether a survey participant's provider was aware of the conditions when considering which counseling to provide. In general, uncontrolled confounding is likely to exist because information on family history and other relevant information that may be accessible to a healthcare provider in a clinical setting was not available. The nature or type of PA medical advice received, or the type or characteristics of the healthcare provider offering PA advice, was not assessed. Finally, limited sample size may have reduced power to observe certain associations.

\section{CONCLUSIONS}

As national objectives encourage healthcare professionals to increase rates of PA medical advice, ${ }^{1-3}$ this study found that the prevalence of receipt of PA medical advice was highest among adults with certain chronic health conditions, including obesity, hypertension, diabetes, hypercholesterolemia, and low HDL cholesterol, and these patterns were stronger for younger adults for diabetes and hypercholesterolemia. However, approximately $57 \%$ of U.S. health care-utilizing adults did not receive medical advice to increase PA in 20132016, indicating adherence to national recommendations for counseling from healthcare providers on PA may not be optimal. ${ }^{1-9}$ The findings may inform public health efforts and initiatives related to the promotion of PA advice to patients by healthcare providers. ${ }^{31,43,45}$ 


\section{Supplementary Material}

Refer to Web version on PubMed Central for supplementary material.

\section{ACKNOWLEDGMENTS}

The National Heart, Lung, and Blood Institute, NIH, Intra-Agency Agreement number A-HL-17-001 provided
funding for select measures described in this paper.

This work was initiated while Dr. Kit was at the Centers for Disease Control and Prevention. The findings and conclusions in this report are those of the authors and not necessarily the official position of the National Center for Health Statistics, Centers for Disease Control and Prevention; National Heart, Lung, and Blood Institute; NIH; or HHS.

\section{REFERENCES}

1. HHS. Healthy People 2020 Physical Activity Objectives. www.healthy-people.gov/2020/ topicsobjectives2020/objectiveslist.aspx?topicId=33. Published 2015 Accessed January 11, 2019.

2. National Physical Activity Plan. U.S. National Physical Activity Plan. http:// physicalactivityplan.org/docs/2016NPAP_Finalforwebsite.pdf. Published 2016 Accessed January 23, 2019.

3. Jacobson DM, Strohecker L, Compton MT, Katz DL. Physical activity counseling in the adult primary care setting: position statement of the American College of Preventive Medicine. Am J Prev Med. 2005;29 (2):158-162. 10.1016/j.amepre.2005.04.009.

4. LeFevre M, U.S. Preventive Services Task Force. Behavioral counseling to promote a healthful diet and physical activity for cardiovascular disease prevention in adults with cardiovascular risk factors: U.S. Preventive Services Task Force recommendation statement. Ann Intern Med. 2014;161(8):587594. 10.7326/M14-1796. [PubMed: 25155419]

5. Patrick K, Pratt M, Sallis R. The healthcare sector's role in the U.S. National Physical Activity Plan. J Phys Act Health. 2009;6(suppl 2):S211-S219. 10.1123/jpah.6.s2.s211.

6. Chodzko-Zajko WJ, Proctor DN, Fiatarone Singh MA, et al. Exercise and physical activity for older adults. Med Sci Sports Exerc. 2009;41(7):1510-1530. 10.1249/MSS.0b013e3181a0c95c. [PubMed: 19516148]

7. Thompson PD, Buchner D, Pina IL, et al. Exercise and physical activity in the prevention and treatment of atherosclerotic cardiovascular disease: a statement from the Council on Clinical Cardiology (Subcommittee on Exercise, Rehabilitation, and Prevention) and the Council on Nutrition, Physical Activity, and Metabolism (Subcommittee on Physical Activity). Circulation. 2003;107(24):3109-3116. 10.1161/01.CIR.0000075572.40158.77. [PubMed: 12821592]

8. Garber CE, Blissmer B, Deschenes MR, et al. Quantity and quality of exercise for developing and maintaining cardiorespiratory, musculo-skeletal, and neuromotor fitness in apparently healthy adults: guidance for prescribing exercise. Med Sci Sports Exerc. 2011;43(7):1334-1359. 10.1249/ MSS.0b013e318213fefb. [PubMed: 21694556]

9. Meriwether RA, Lee JA, Lafleur AS, Wiseman P. Physical activity counseling. Am Fam Physician. 2008;77(8):1129-1136. [PubMed: 18481560]

10. Kreuter M, Scharff D, Brennan L, Lukwago SN. Physician recommendations for diet and physical activity: which patients get advised to change? Prev Med. 1997;26(6):825-833. 10.1006/ pmed.1997.0216. [PubMed: 9388794]

11. Wee C, McCarthy EP, Davis RB, Phillips RS. Physician counseling about exercise. J Am Med Assoc. 1999;282(16):1583-1588. 10.1001/jama.282.16.1583.

12. Glasgow RE, Eakin EG, Fisher EB, Bacak SJ, Brownson RC. Physician advice and support for physical activity: results from a national survey. Am J Prev Med. 2001;21(3):189-196. 10.1016/ S0749-3797(01)00350-6. [PubMed: 11567839]

13. Barnes P, Schoenborn C. Trends in adults receiving a recommendation for exercise or other physical activity from a physician or other health professional. NCHS Data Brief. 2012;86:1-8. 
14. National Center for Health Statistics. NHANES Response Rates and Population Totals. wwwn.cdc.gov/Nchs/Nhanes/ResponseRates.aspx. Published 2017 Accessed January 24, 2019.

15. CDC. National Health and Nutrition Examination Surveys: NHANES 2011-2012. wwwn.cdc.gov/ Nchs/Nhanes/ContinuousNhanes/Default.aspx?Begin Year=2011. Published 2012 Accessed February 9, 2017.

16. CDC. National Health and Nutrition Examination Surveys: NHANES 2013-2014. wwwn.cdc.gov/ Nchs/Nhanes/ContinuousNhanes/Default.aspx?Begin Year=2013. Published 2014 Accessed February 9, 2017.

17. CDC. National Health and Nutrition Examination Survey, 2011-2012 Medical Conditions Questionnaire Documentation. https://wwwn.cdc.gov/nchs/nhanes/2011-2012/MCQ_G.htm. Accessed January 1, 2019.

18. CDC. National Health and Nutrition Examination Survey, 2013-2014 Medical Conditions Questionnaire Documentation. https://wwwn.cdc.gov/Nchs/Nhanes/2013-2014/MCQ_H.htm. Accessed January 1, 2019.

19. HHS. Physical Activity and Health: A Report of the Surgeon General. Atlanta, GA: CDC; 1996.

20. Bakris GL, Black HR, Cushman WC, et al. The Seventh Report of the Joint National Committee on Prevention, Detection, Evaluation, and Treatment. JAMA. 2015;289(19):2560-2573. 10.1001/ jama.289.19.2560.

21. Physical Activity Guidelines Advisory Committee. Physical Activity Guidelines Advisory Committee Report. www.health.gov/paguidelines/pdf/paguide.pdf. Published 2008 Accessed January 11, 2019.

22. Vaccaro JA, Huffman FG. Relationship of lifestyle medical advice and non-HDL cholesterol control of a nationally representative U.S. sample with hypercholesterolemia by race/ethnicity. Cholesterol. 2012;2012:916816 10.1155/2012/916816. [PubMed: 23119150]

23. Vaccaro JA, Huffman FG. Gender differences in medical advice and health behavior of obese African Americans with and without type 2 diabetes. Am J Mens Health. 2012;6(5):383-394. 10.1177/1557988312449853. [PubMed: 22691306]

24. Vaccaro JA, Feaster DJ, Lobar SL, Baum MK, Magnus M, Huffman FG. Medical advice and diabetes self-management reported by Mexican-American, black- and white-non-Hispanic adults across the United States. BMC Public Health. 2012;12:185 10.1186/1471-2458-12-185. [PubMed: 22410191]

25. CDC, National Center for Health Statistics. National Health and Nutrition Examination Survey: Analytic Guidelines, 2011-2012 Hyattsville, MD: CDC, National Center for Health Statistics, Division of Health and Nutrition Examination Surveys. wwwn.cdc.gov/nchs/data/nhanes/ 2011-2012/analytic_guidelines_11_12.pdf. Published 2013 Accessed January 11, 2019.

26. Lobelo F, Young DR, Sallis R, et al. Routine assessment and promotion of physical activity in healthcare settings: a scientific statement from the American Heart Association. Circulation. 2018;137(18):e495-e522. 10.1161/CIR.0000000000000559. [PubMed: 29618598]

27. Albright CL, Cohen S, Gibbons L, et al. Incorporating physical activity advice into primary care physician-delivered advice within the Activity Counseling Trial. Am J Prev Med. 2000;18(3):225234. 10.1016/S0749-3797(99)00155-5. [PubMed: 10722989]

28. Bull FC, Jamrozik K. Advice on exercise from a family physician can help sedentary patients to become active. Am J Prev Med. 1998;15(2):85-94. 10.1016/S0749-3797(98)00040-3. [PubMed: 9713663]

29. Calfas K, Long B, Sallis J, Wooten W, Pratt M, Patrick K. A controlled trial of physician counseling to promote the adoption of physical activity. Prev Med. 1996;25(3):225-233. 10.1006/ pmed.1996.0050. [PubMed: 8780999]

30. Lewis B, Lynch W. The effect of physician advice on exercise behavior. Prev Med. 1993;22(1):110-121. 10.1006/pmed.1993.1008. [PubMed: 8475007]

31. Heath GW, Kolade VO, Haynes JW. Exercise is Medicine ${ }^{\mathrm{TM}}$ : a pilot study linking primary care with community physical activity support. Prev Med Rep. 2015;2:492-497. 10.1016/ j.pmedr.2015.06.004. [PubMed: 26844108] 
32. Jimmy G, Winfried BM. Implementation and effectiveness of a primary care based physical activity counselling scheme. Patient Educ Couns. 2005;56(3):323-331. 10.1016/j.pec.2004.03.006. [PubMed: 15721975]

33. Pinto B, Goldstein M, Marcus B. Activity counseling by primary care physicians. Prev Med. 1998;513(27):506-513. 10.1006/pmed.1998.0335.

34. Petrella R, Koval J, Cunningham D, Paterson D. Can primary care doctors prescribe exercise to improve fitness? The Step Test Exercise Prescription (STEP) project. J Prev Med. 2003;24(4):316322. 10.1016/S0749-3797(03)00022-9.

35. Tulloch H, Fortier M, Hogg W. Physical activity counseling in primary care: who has and who should be counseling? Patient Educ Couns. 2006;64(1-3):6-20. 10.1016/j.pec.2005.10.010. [PubMed: 16472959]

36. Forjuoh SN, Lee C, Won J, Towne SD Jr, Wang S, Ory MG. Correlates of receiving a recommendation for more physical activity from a primary care provider. Am J Prev Med. 2017;52(2):207-214. 10.1016/j.amepre.2016.09.037. [PubMed: 27890517]

37. Bauman AE, Reis RS, Sallis JF, Wells JC, Loos RJF, Martin BW. Correlates of physical activity: why are some people physically active and others not? Lancet. 2012;380(9838):258-271. 10.1016/ S0140-6736(12)60735-1. [PubMed: 22818938]

38. Giles-Corti B, Donovan R. The relative influence of individual, social and physical environment determinants of physical activity. Soc Sci Med. 2002;54(12):1793-1812. 10.1016/ S0277-9536(01)00150-2. [PubMed: 12113436]

39. Patnode C, Evans C, Senger C, Redmond N, Lin J. Behavioral counseling to promote a healthful diet and physical activity for cardiovascular disease prevention in adults without cardiovascular risk factors: U.S. Preventive Services Task Force recommendation statement. JAMA. 2017;318(2):167-174. 10.1001/jama.2017.3303. [PubMed: 28697260]

40. Joy E, Blair S, McBride P, Sallis R. Physical activity counselling in sports medicine: a call to action. Br J Sports Med. 2013;47(1):49-53. 10.1136/bjsports-2012-091620. [PubMed: 23149653]

41. Moyer V, U.S. Preventive Services Task Force. Behavioral counseling interventions to promote a healthful diet and physical activity for cardiovascular disease prevention in adults: U.S. Preventive Services Task Force recommendation statement. Ann Intern Med. 2012;161(8):587-594. 10.7326/0003-4819-157-5-201209040-00486.

42. Artinian NT, Fletcher GF, Mozaffarian D, et al. Interventions to promote physical activity and dietary lifestyle changes for cardiovascular risk factor reduction in adults: a scientific statement from the American Heart Association. Circulation. 2010;122(4):406-441. 10.1161/ CIR.0b013e3181e8edf1. [PubMed: 20625115]

43. Lobelo F, Stoutenberg M, Hutber A. The exercise is medicine global health initiative: a 2014 update. Br J Sports Med. 2014;48(22):1627-1633. 10.1136/bjsports-2013-093080. [PubMed: 24759911]

44. Tucker J, Welk G, Beyler N. Physical activity in U.S.: adults compliance with the Physical Activity Guidelines for Americans. Am J Prev Med. 2011;40(4):454-461. 10.1016/j.amepre.2010.12.016. [PubMed: 21406280]

45. National Park Service. Park RX. www.parkrx.org/. Accessed January 11, 2019. 
Adults $\geq 20$ years interviewed and examined in 2013-2016 $n=11,062$

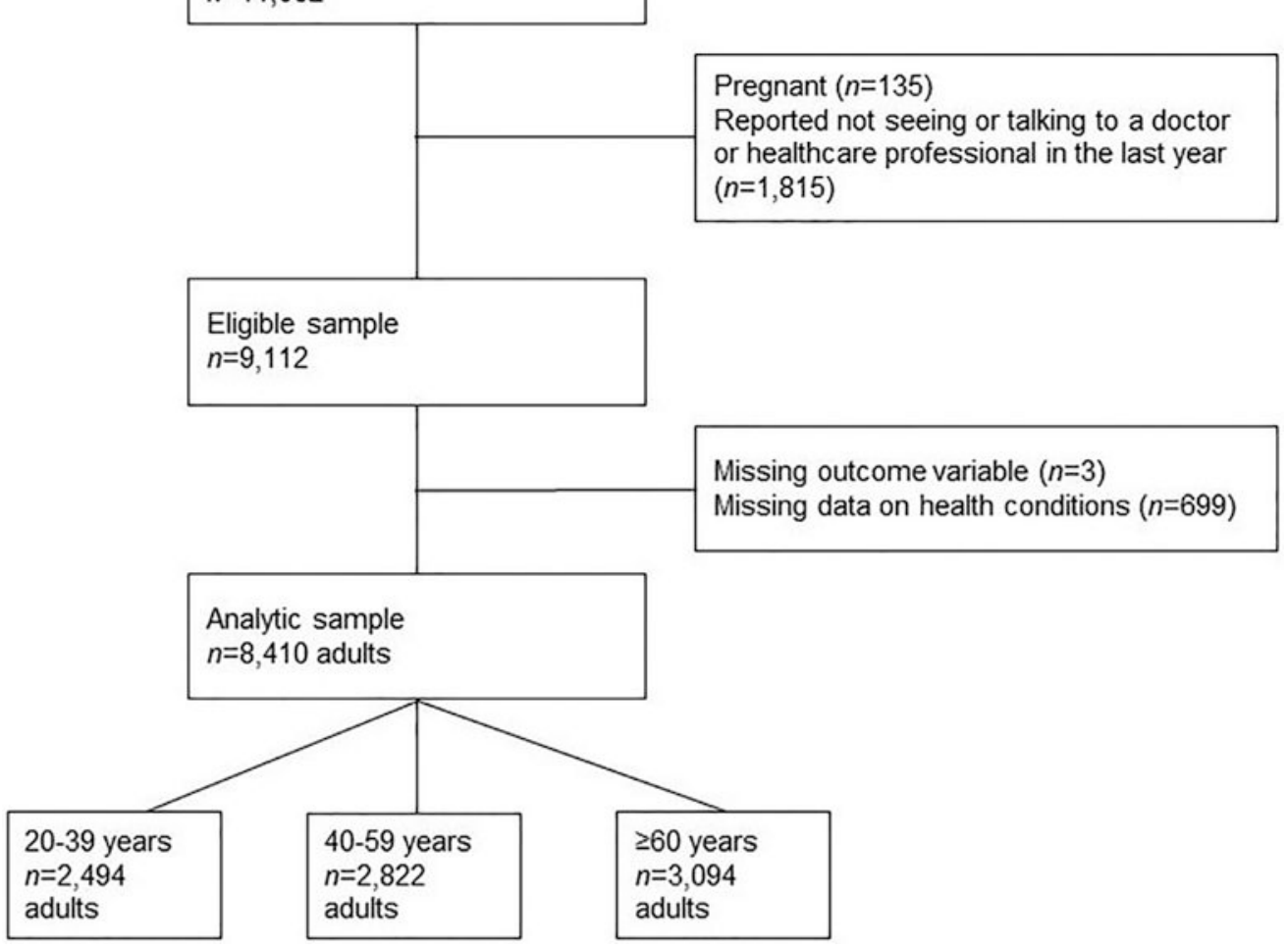

Figure 1.

Analytic sample. 


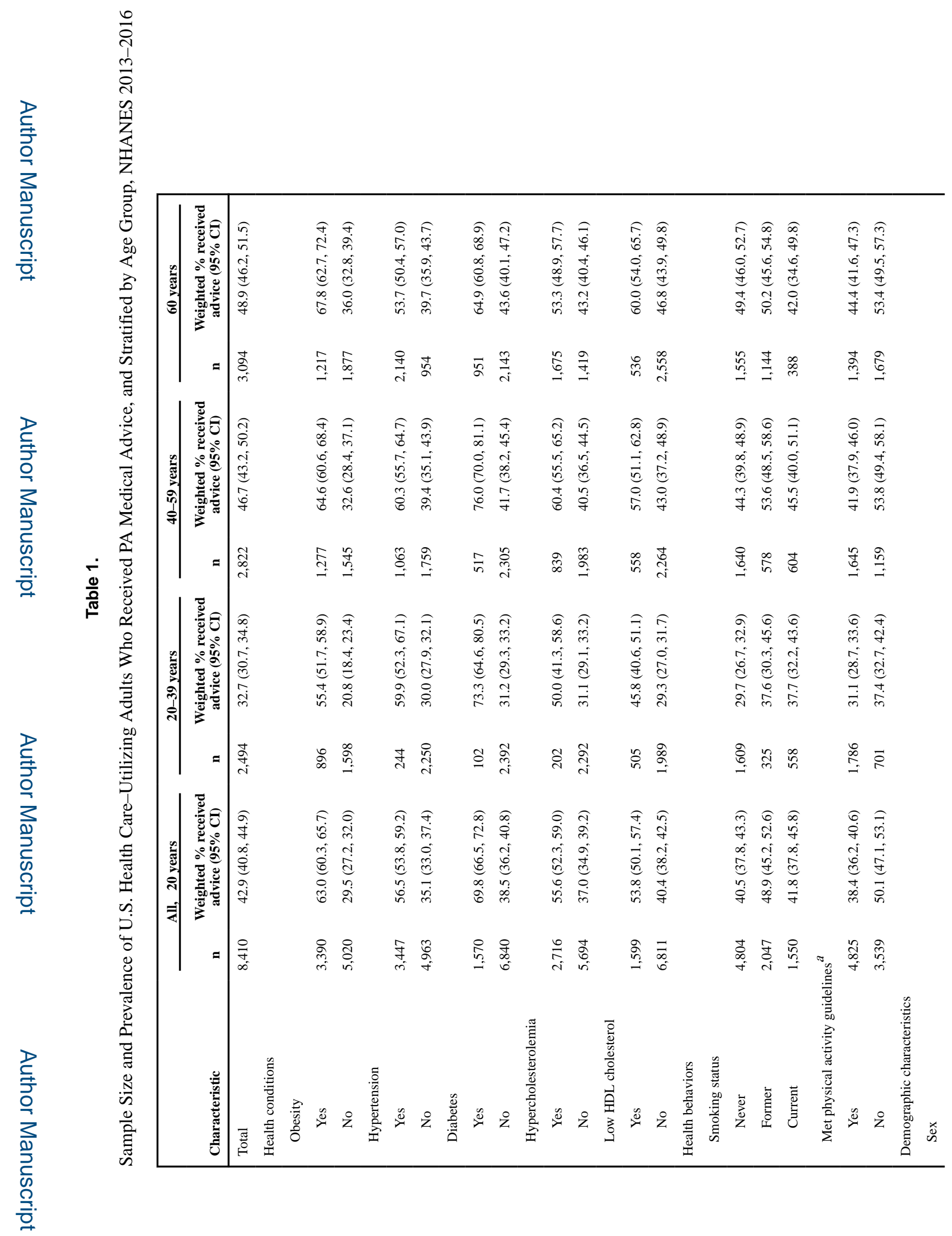

Am J Prev Med. Author manuscript; available in PMC 2020 May 13. 


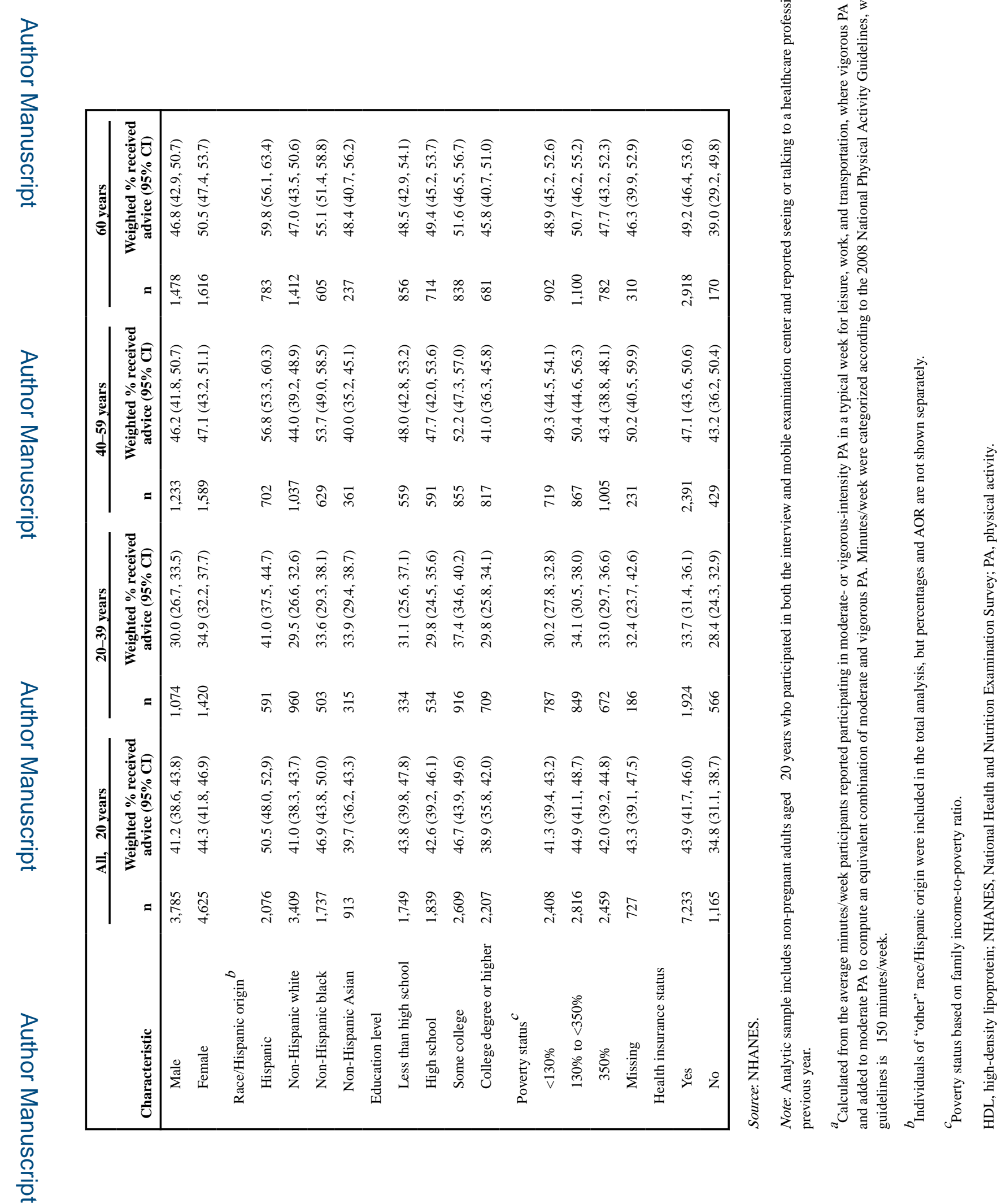




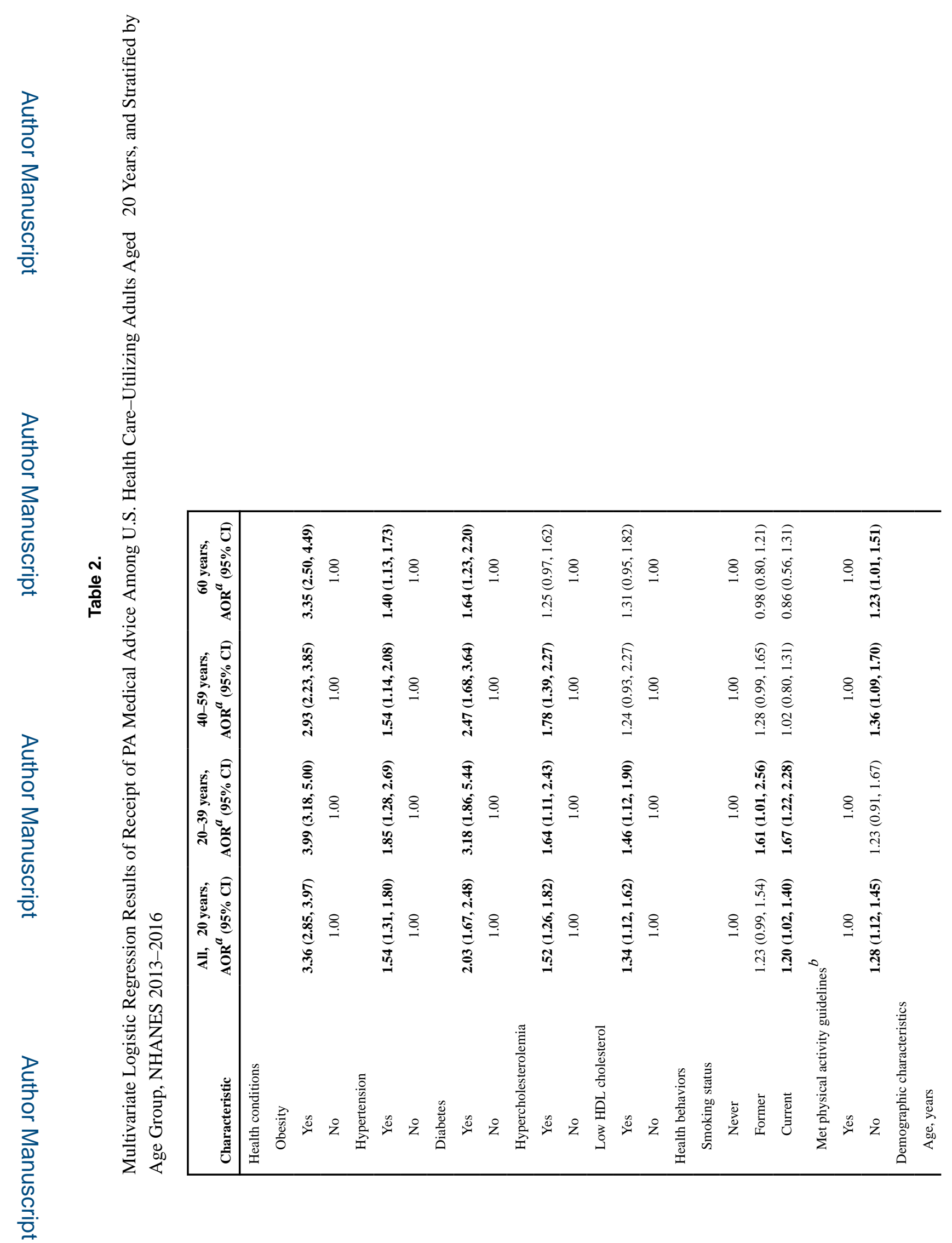

Am J Prev Med. Author manuscript; available in PMC 2020 May 13. 


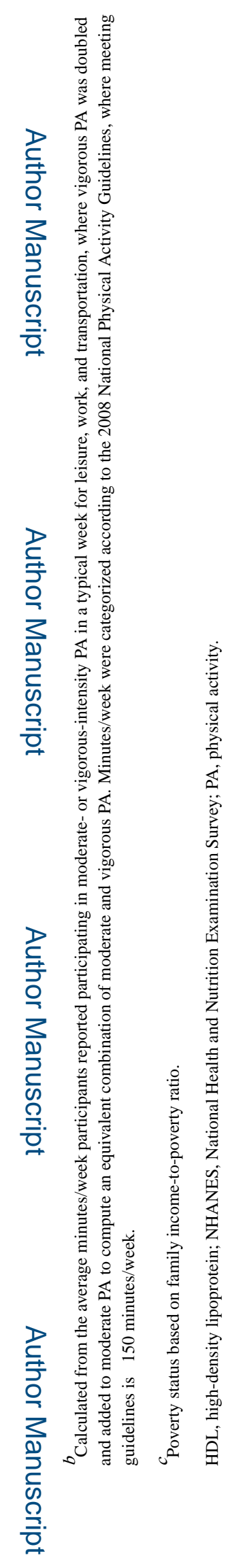

Am J Prev Med. Author manuscript; available in PMC 2020 May 13. 